\title{
L'(in)sécurisation par les technologies militaires et la mise en sens de la violence
}

Le cas de l'intervention militaire de l'OTAN au-dessus de la Libye (2011)

The (in)securisation by military technologies and the significance of violence: a case-study of the NATO military intervention in Libya (2011)

\section{Julien Pomarède}

\section{(2) OpenEdition}

\section{Journals}

\section{Édition électronique}

URL : http://journals.openedition.org/conflits/18867

DOI : 10.4000/conflits. 18867

ISSN : 1777-5345

Éditeur :

CCLS - Centre d'études sur les conflits lilberté et sécurité, L'Harmattan

\section{Édition imprimée}

Date de publication : 8 juillet 2014

Pagination : 125-145

ISBN : 978-2-343-04155-1

ISSN : 1157-996X

Référence électronique

Julien Pomarède, «L'(in)sécurisation par les technologies militaires et la mise en sens de la violence », Cultures \& Conflits [En ligne], 93 | printemps 2014, mis en ligne le 02 juillet 2015, consulté le 30 mars 2021. URL : http://journals.openedition.org/conflits/18867; DOI : https://doi.org/10.4000/conflits. 18867 


\title{
L'(in)sécurisation
}

\section{par les technologies militaires et la mise en sens de la violence :}

\author{
Le cas de l'intervention militaire de l'OTAN \\ au-dessus de la Libye (2011) 1
}

\section{Julien POMAREDE}

Julien Pomarède vient d'achever un Master en Science politique, mention Relations internationales, à l'Université Libre de Bruxelles (ULB). Il est actuellement assistant d'enseignement à la Faculté des Sciences politiques de l'ULB et entame une thèse de doctorat en science politique/relations internationales sur l'OTAN auprès du centre Recherche et enseignement en politique internationale (REPI).

Se déroulant dans le contexte de la révolution libyenne qui débuta en janvier 2011, l'intervention militaire de l'OTAN dans le ciel libyen fut déclenchée suite au vote de la résolution 1973 du Conseil de sécurité des Nations unies qui autorisait les États membres à prendre «toutes les mesures nécessaires [...] pour protéger les populations et zones civiles menacées d'attaque en Jamahiriya arabe libyenne 2 ». Baptisée Unified Protector, cette opération, en tête de laquelle se situaient la France, la Grande-Bretagne et les États-Unis, débuta officiellement le 31 mars 2011 pour s'achever le 31 octobre de la même année. L'enjeu de cet article ne sera pas de s'insérer dans le débat ayant eu lieu autour du dépassement ou non du mandat onusien par l'OTAN. Celui-ci prévoyait l'objectif limité de la protection des populations et ne nommait pas explicitement la menace contre laquelle les civils devaient être protégés, ce qui conduisit l'OTAN à mener une campagne aérienne sans pouvoir désigner d'ennemi. Les commentaires autour de l'intervention ont alors principalement

1. Cet article est issu d'un travail de mémoire de Master et a fait l'objet d'une première présentation lors du colloque Techniques and Technologies of Security in International Relations à l'Université Libre de Bruxelles en avril 2013. Nous tenons à remercier vivement Julien Canin, Barbara Delcourt, Julien Jeandesboz, Erika Luzzato, Christian Olsson et Christophe Wasinski ainsi que les évaluateurs anonymes pour leurs critiques constructives sur la version préliminaire de cet article.

2. Conseil de sécurité des Nations unies, Résolution 1973 du 17 mars 2011, \$4. 
porté sur l'ambiguïté autour du ciblage des bombardements : Kadhafi et ses partisans étaient-ils considérés comme un ennemi et une cible en soi ? Une question souvent associée à des critiques ${ }^{3}$ auxquelles l'OTAN et ses membres répondaient qu'ils ne faisaient qu'appliquer à la lettre ("no more no less ») les termes de la résolution $1973{ }^{4}$.

Cette étude ne portera pas sur cette polémique, mais se conçoit comme une analyse de la manière dont les problèmes de sécurité sont construits et représentés dans les organisations internationales, comme l'OTAN, qui visent à assurer et gérer la sécurité internationale. Nous emprunterons ainsi la notion d'(in)sécurisation développée notamment dans les travaux de Didier Bigo, qui considère que sécurité et insécurité font l'objet de représentations qui vont de pair en se dynamisant mutuellement. Son analyse du processus de légitimation des professionnels de la sécurité révèle que l'affirmation de leur compétence à assurer la sécurité passe par la représentation des insécurités ${ }^{5}$. Par opposition à la securitization theory ${ }^{6}$ qui sépare les deux termes en expliquant qu'une insécurité n'est plus considérée comme telle lorsque la situation se retrouve reformulée en termes de sécurité, les deux termes se trouvent intriqués, la création d'une certaine image des inquiétudes, de ce dont il faut avoir peur (les insécurités), conditionnant la possibilité pour ces professionnels d'affirmer leur capacité à les gérer, les régler. En d'autres termes, les moyens et savoirfaire produits par ces acteurs en vue de « combattre » les insécurités contribuent dans le même temps à en produire la représentation. Pour résumer, dire ce qu'est la sécurité passe par dire ce dont on doit avoir peur, si bien que «souvent, quand une (in)sécurisation est produite, sécurité et insécurité croissent ensemble, générant ainsi une dynamique autosuffisante ${ }^{7}$ ».

L’opération militaire en Libye sera considérée comme un moment particulièrement visible au cours duquel l'OTAN formula une définition particulière de l'(in)sécurité dans l'optique de pérenniser sa légitimité en tant qu'organisme central dans la gestion des risques et menaces internationaux. Dans cette perspective, la pertinence de l'opération militaire dans le ciel libyen relève de deux raisons.

3. Pour une analyse critique, voir : Andersson N., Lagot D. (eds.), Responsabilité de protéger et guerres " humanitaires ": le cas de la Libye, Harmattan, Paris, 2012, 155 pages.

4. Lungescu O., Press briefing on events related to Libya by LtGen Charles Bouchard, Commander of the NATO military operations in Libya and NATO Spokesperson Oana Lungescu, 28 March 2011 ; Rasmussen A. F., NATO Secretary General's statement on Libya no-fly zone, 24 March 2011.

5. Bigo D., « La mondialisation de l'(in)sécurité ? Réflexions sur le champ des professionnels de la gestion des inquiétudes et analytique de la transnationalisation des processus d'(in)sécurisation ", Cultures E Conflits, 58, 2005, pp. 53-101.

6. Waever O., "Securitization and Desecuritization", in Lipschutz R. (ed.), On Security, Colombia University Press, New-York, 1995, pp. 46-86.

7. Bigo D., "International Political Sociology”, in Williams P. (ed.), Security Studies, An introduction, Routledge, 2008, New York, p. 124. 
La première concerne le lien entre emploi de la violence et (in)sécurité. Comme nous le verrons, l'état d'insécurité généré par Kadhafi en Libye est inscrit dans un discours otanien dont le narratif se construit autour de l'usage de la violence. Pour contrer cette violence dirigée contre les civils et y mettre fin, l'Alliance employa elle-même une violence, à travers les bombardements, qui est apparue comme «plus légitime » et sécurisante que celle de Kadhafi. Le premier type de questionnement qui se pose à travers ce cas d'étude est donc le suivant : pourquoi l'exercice d'une violence paraît-il plus «normal », "légitime » et sécurisant qu'un autre ? Quelles sont les pratiques (ici militaires) ainsi que leurs insertions dans les contenus normatifs qui permettent de rattacher telle ou telle forme de violence à une situation d'(in)sécurité ? La gestion concrète, les procédés routinisés de l'exercice du bombardement participent-ils à la construction de ces représentations ?

La seconde raison relève de la présence surabondante du registre technique dans la communication externe de l'OTAN durant l'intervention. Par opposition au contenu très idéologique des déclarations des personnalités officielles des États membres de l'OTAN, les discours des représentants politiques et militaires de l'institution otanienne étaient souvent composés de très longs récits techniques qui expliquaient en détails la manière dont l'intervention était menée. Le Secrétaire général, Anders Fogh Rasmussen, s'exprima d'ailleurs assez peu par comparaison aux responsables militaires qui furent souvent invités pour exposer de manière détaillée le déroulement concret des opérations. Il nous paraît donc intéressant de nous pencher sur ce cas d'étude afin de comprendre comment les problèmes de sécurité peuvent être représentés à partir d'une référence à certaines techniques et technologies militaires : une mise en sens pratique et discursive de la violence en apparence éthérée et descriptive n'est-t-elle qu'une simple description de la réalité ou est-t-elle porteuse d'une interprétation qui vise à fournir une version (il)légitime et (in)acceptable de la réalité ? Un acte qui apparaît aussi anodin et courant qu'un compte rendu public d'opération par un officier militaire ne serait-il pas en lui-même porteur d'une représentation de ce qu'est la définition de la sécurité, du risque ou de la menace ? À travers ce cas d'étude, nous souhaitons donc nous écarter de l'étude des procédés d'(in)sécurisation contenus dans les déclarations à forte consonances idéologiques, afin de nous plonger dans ceux qui émanent de pratiques militaires routinisées, peu visibles aux yeux du grand public, mais qui participent très concrètement à la construction des imaginaires d'(in)sécurité. 


\section{Théoriser l'OTAN : de la problématique de l'existence de l'Alliance à celle des (in)sécurisations}

Le débat académique sur l'OTAN a été marqué durant ces vingt dernières années par cette question : "pourquoi l'OTAN se maintient-elle après la Guerre froide ? » Très souvent interprétée comme un moment crucial de l'histoire de l'OTAN, la fin du système des blocs et la chute de l'adversaire qui avait motivé la création de l'Alliance en 1949 constitue le point de référence de la plupart des études contemporaines sur l'OTAN. Trois grands courants théoriques ont fournit les explications les plus reprises dans la littérature sur le sujet. Le premier est le néoréalisme, pour lequel une alliance ne représente qu'une réponse rationnelle des États membres face à une menace dite objective ${ }^{8}$. Cette dernière est alors considérée comme un fait possédant une essence propre, indépendante de la subjectivité des acteurs qui y font face, et dont la plus ou moins grande prégnance qui s'impose à eux les oblige à adopter les moyens les plus efficaces pour y répondre. L'existence de l'OTAN est estimée comme menacée depuis la fin de la Guerre froide en raison de la chute de son ennemi fondateur, l'URSS 9. Le contexte d'anarchie expliquerait la pérennité de l'OTAN qui serait un moyen pour les grandes puissances de préserver une hégémonie sur les puissances inférieures membres de l'Alliance. Le second est le néolibéralisme institutionnel, qui considère l'OTAN non plus comme un agrégat de politiques de sécurité nationale égoïstes au service de la survie individuelle des États la composant, mais comme un espace de coopération interétatique en matière de sécurité qui permettrait d'assurer une certaine stabilité à l'échelle internationale. Le maintien de l'Alliance relèverait ainsi de sa capacité d'adaptation institutionnelle à un environnement considéré comme en mutation. La persistance de l'OTAN s'expliquerait par le fait que le coût de sa dissolution pour les États membres serait bien plus élevé que celui de son maintien et de son adaptation à un environnement de sécurité post-bipolaire où de nouvelles menaces se feraient jour ${ }^{10}$. Celles-ci exigeraient ainsi de nouvelles formes de coopération au sein d'une Alliance qui aurait fait ses preuves en termes de gestion des risques internationaux. Le troisième courant, le constructivisme conventionnel, se concentre sur l'implication des identités et des normes dans les dynamiques internationales. Les tenants de ce courant établissent en effet que les intérêts qui guident les acteurs sur la scène internationale sont façonnés par leurs identités et le contexte normatif dans lequel ils

8. McCalla R., "NATO's persistence after the Cold War", International Organization, 50-3, 1996, p. 447 ; Walt S., "Alliance Formation and the Balance of World Power", International Security, 9-4, 1985, p. 29 ; Waltz K., Theory of International Politics, Addison-Wesley Publishing, Menlo Park, 1979, pp. 169-170.

9. Mearsheimer J., "Back to the Future. Instability in Europe after the Cold War", International Security, 15-1, 1990, pp. 5-56 ; Waltz K., "The Emerging Structure of International Politics", International Security, 18-2, 1993, pp. 44-79.

10. Duffield J., "NATO's Functions after the Cold War", Political Science Quarterly, 105-5, 1994-1995, p. 766 ; Wallander C., "Institutional Assets and Adaptability: NATO after the Cold war”, International Organization, 54-4, 2000, p. 705. 
se trouvent plongés ${ }^{11}$. Dans cette perspective, l'OTAN ne serait pas l'expression d'une réponse rationnelle face à une menace ou le fait d'une inertie institutionnelle, mais correspondrait à une « communauté de sécurité » dont l'essence est le partage par les États membres d'une identité libérale-démocratique ${ }^{12}$. L'espace nord-atlantique serait caractérisé par un sentiment commun d'appartenance si élevé qu'il rendrait la guerre impensable entre les États membres de la communauté de sécurité ${ }^{13}$. Ce courant théorique conçoit cette homogénéité identitaire comme très stable et allant de soi, son but n'étant pas d'explorer les modalités de formation de cette identité. Il s'agit d'une variable explicative qui est utilisée pour montrer en quoi le partage d'une identité collective peut faciliter « l'émergence d'institutions de coopération dans certains domaines »14. Le problème commun à ces approches est qu'elles conçoivent les contextes internationaux de sécurité comme des situations objectives dans lesquelles l'OTAN est insérée. Qu'il soit anarchique, en mutation ou stabilisé et pacifique, l'environnement international dans lequel évolue l'OTAN est donc pris comme acquis, et n'est pas remis en question dans la perspective de la manière dont cet acteur peut en façonner la représentation dans l'optique de légitimer un statut clef dans la gestion des insécurités.

Notre démarche ne s'inscrit pas dans ce questionnement du pourquoi de la pérennité de l'Alliance. Cet article n'a pas pour but une tentative de construire une nouvelle théorie générale sur le maintien de l'OTAN, mais de l'étudier sous l'angle d'une sociologie politique de l'international, en nous interrogeant sur la manière dont les problèmes de sécurité sont représentés et façonnés dans cette organisation internationale. L'avantage d'une telle démarche est qu'elle nous permettra, contrairement aux approches mentionnées précédemment, de dé-substantialiser les environnements d'(in)sécurité qu'un organisme comme l'OTAN vise à gérer. Les représentations dont ils font l'objet ne seront pas considérées comme des situations objectives, qui émergeraient par elles-mêmes, qui possèderaient une existence pré-empirique en dehors de toute implication pratique et discursive de l'acteur qui prétend y répondre par «l'évidence » de sa compétence. En adaptant une démarche constructiviste critique, nous souhaitons au contraire nous pencher sur les pratiques et les discours d'(in)sécurisation établis au sein d'un des organismes impliqués dans la gestion des risques et menaces internationaux. Nous postulons donc que cette capacité est une évidence qui «ne va pas de soi ». Elle se trouve notamment constituée par le biais de la production d'un savoir-faire

11. Wendt A., Social Theory of International Politics, Cambridge University Press, Cambridge, 1999, p. 1.

12. Adler E., Barnett M., Security communities, Cambridge University Press, Cambridge, 1998, p. 51.

13. Deutsch K., Political community and the North Atlantic area: international organization in the light of historical experience, Princeton University Press, Princeton, 1957, p. 5.

14. Risse-Kappen T., "Collective Identity in a Democratic Community: The Case of NATO", in Katzenstein P. (ed.), The Culture of National Security: Norms and Identity in World Politics, Colombia University Press, New-York, 1996, p. 397. 
technique tourné vers l'utilisation et la gestion de la violence armée, prenant source dans des pratiques routinisées et des discours à partir desquels l'OTAN bâtit et valide sa légitimité en tant qu'acteur assurant la sécurité internationale. C'est dans cette perspective que nous allons étudier l'opération de l'OTAN dans le ciel libyen ${ }^{15}$. En analysant les technologies militaires déployées et les discours produits lors de cette intervention, nous allons tâcher de comprendre comment les inquiétudes et insécurités internationales sont façonnées et représentées au sein de l'Alliance à travers ces vecteurs de sens.

\section{Le schéma libyen : l'(in)sécurisation par l'(ir)rationalité dans l'emploi de la violence}

\section{Le référent de la violence}

Durant l'intervention militaire en Libye, les procédés pratiques et discursifs d'(in)sécurisation s'organisèrent autour d'un référentiel particulier, celui de la violence. Pour mieux comprendre l'importance de la place de ce référent, nous allons l'aborder à travers deux concepts méthodologiques empruntés à Michel Foucault. Le premier est l'« objet du discours ». Il s'agit du point d'articulation à partir duquel le discours et les technologies se développent ${ }^{16}$. Il peut être considéré comme ce dont le discours "parle », ce en fonction de quoi les technologies sont employées. Comme le note Foucault à propos de la psychiatrie, l'objet du discours et des pratiques qui s'y rapportent est la folie ${ }^{17}$. C'est autour de ce référent qu'ils se construisent. L'objet organise les « grilles de spécification », qui sont « des systèmes selon lesquels on sépare, on oppose, on apparente, on regroupe, on classe, [...] les unes des autres les différentes formes de “folies" comme objets du discours psychiatrique 18 ». Si l'objet du discours et des pratiques est le point névralgique autour duquel ces derniers s’établissent, il est aussi le prisme par lequel se catégorise la réalité. L’objet sur lequel se focalisent le discours et les technologies est donc la source essentielle de la catégorisation de la réalité. C’est par lui que la différenciation entre sujets du monde social se crée.

15. Pour autant, notre démarche ne consiste pas à nier l'existence et l'impact de la violence physique réelle déployée par l'OTAN ou le régime libyen, ni à juger et évaluer la légitimité de l'emploi de la violence par les acteurs en présence. Loin de nous l'idée de minimiser les conséquences d'une violence qui furent bien réelles pour les populations concernées, comme l'attestent plusieurs rapports produits sur cette question (Cour pénale internationale, Premier rapport du procureur de la Cour pénale internationale au Conseil de sécurité des Nations unies en application de la résolution 1970 (2011), 4 mai 2011 ; Human Rights Watch, Unacknowledged Deaths: Civilians Casualties in NATO's Air Campaign in Libya, 2012). Notre réflexion porte bel et bien sur la mise en sens de cette violence (qui a été effectivement déployée) et la manière dont sa représentation dans les pratiques et discours tendent à (dé)légitimer les acteurs qui en font emploi.

16. Foucault M., L'archéologie du savoir, Gallimard, Paris, 1969, p. 55.

17. Ibid., p. 56.

18. Ibid., p. 58 ; Foucault M., Histoire de la folie à l'âge classique, Gallimard, Paris, 1976, p. 132. 
Dans le cas de l'intervention militaire en Libye, le discours et l'emploi des technologies militaires prennent appui sur l'objet de la violence. Ce registre est très présent au moment de l'opération. Alors que le Conseil de sécurité autorisa le recours à la force en raison de l'emploi de la violence contre les civils, l'OTAN usa des technologies militaires et de la violence pour protéger les civils contre les violences qui leur furent faites. La violence organisa également les grilles de spécification qui se déployèrent par une catégorisation de la réalité selon une différenciation entre un Soi légitime et sécurisant et un Autre illégitime et menaçant. Kadhafi était en effet condamné par les parties intervenantes et n'était plus perçu comme le gouvernant légitime en raison de son emploi excessif de la violence. Comme l'exprima notamment Hillary Clinton, «Nous avons vu les forces de sécurité du Colonel Kadhafi ouvrir le feu sur des manifestants pacifiques... Par ces actions, les dirigeants libyens ont perdu la légitimité nécessaire pour gouverner. [...] Il est temps pour Kadhafi de partir, maintenant, sans autre violence ou délai 19 ». À l'inverse, et comme nous le verrons en détails, l'intervention de l'OTAN fut représentée comme légitime et sécurisante en raison de son usage instrumental-rationnel de la violence. Le référentiel de la violence fut ainsi le point névralgique à partir duquel s'opérèrent les procédés d'(in)sécurisation.

Plus précisément, l'(in)sécurisation consista, dans les discours et l'emploi des technologies militaires, en un portrait du Soi et de l'Autre à travers une capacité intrinsèque à mobiliser la violence sur une base (ir)rationnelle. L'usage rationnel de la violence étant associé durant l'opération à une situation de sécurité (car limité dans le temps, en intensité et lié à des buts précis) tel était le portrait donné par l'OTAN de son emploi de la violence. L'emploi irrationnel de la violence étant rattaché au contraire à une insécurité (car représenté comme illimité, indiscriminé et dépourvu d'objectifs clairs) - telle fut l'image donnée à l'usage de la violence par Kadhafi. La violence fut donc la grille par laquelle un sens de l'(in)sécurité fut constitué, selon une différenciation entre l'utilisation rationnelle de la violence par l'OTAN et le recours irrationnel de la force par Kadhafi.

Les logiques d'(in)sécurisation: «sens commun stratégique » et déshumanisation de la guerre

Quels furent les procédés discursifs et pratiques concrets qui permirent d'affirmer une telle représentation, une telle différenciation? Nous travaillerons autour de deux types de logiques participant à la mise en sens (il)légitime et (in)sécurisante de la violence. La première est celle de la « technologisation » de la guerre.

19. Clinton H., Press Conference, Geneva, 28 February 2011. Nous précisons au passage que l'ensemble des citations extraites de productions en anglais a fait l'objet d'une traduction en français dans le cadre de cet article. 
Selon Christophe Wasinski, les guerres menées par les puissances occidentales sont conduites dans le cadre cognitif d'un « sens commun stratégique » qui « délimiterait la signification et la pratique de ce que l'on entend par guerre. [...] Le régime guerrier serait à la fois un régime d'encadrement plus ou moins efficace de la violence armée et de légitimation du combat $20 »$. Ce sens commun est façonné par une représentation technique de la guerre, si bien qu'il établit que l'usage de « la force militaire est conditionné par une représentation qui fait de celle-ci un moyen technique crédible, rationnel sur le plan instrumental et, partant de là, socialement acceptable 21 ». Il a donc pour but fondamental de fixer la manière dont la violence doit être employée pour paraitre « légitime » dans la conduite de la guerre. Il entreprend une différenciation entre les pratiques violentes qui relèvent du normal et du légitime et celles perçues comme anormales et illégitimes. La représentation technique de la guerre est le moyen clef par lequel la violence est conçue sur un mode rationnel et, in fine, légitime. L'usage de technologies militaires particulièrement sophistiquées permet d'utiliser la violence sur une base toujours plus rationnelle, car la précision associée à ces technologies évite la représentation de l'acte de violence comme excessif et déraisonnable. En d'autres termes, la technologie militaire renforce ainsi le système d'opposition susceptible de se créer par rapport à un Autre qui emploierait la violence de manière irrationnelle.

La seconde logique par laquelle les (in)sécurisations se créent en référence à la violence est la déshumanisation de la guerre, à savoir l'évacuation et la mise sous silence de sa dimension humaine. La souffrance, la peur, le sang, les morts, qui fondent la réalité du phénomène guerre sont passés « sous silence par la grammaire narrative 22 » de la technologisation de la guerre. Ce procédé déshumanisant a pour fonction de solidifier toujours plus la légitimité du mode d'emploi rationnel de la violence et de le rendre socialement acceptable, voire évident, car masquer l'effet physique de la violence sur les corps permet de l'inscrire dans le registre du raisonnable. À l'inverse, dès lors que la guerre est narrée dans la trivialité des membres et des corps déchiquetés, celui qui est rattaché à un tel portrait serait vu comme le déraisonné. Dans une tonalité foucaldienne, nous dirons que ces processus de déshumanisation relèvent d'une «technologie politique des corps ", une extraction du savoir à partir de l'inscription d'un pouvoir sur le corps humain ${ }^{23}$. Les technologies militaires ordonnent un traitement du corps par une esthétique aseptisée, de manière à légitimer la représentation d'une violence de type instrumental-rationnel. Le corps humain peut donc être considéré comme un support fondamental sur

20. Wasinski C., Rendre la guerre possible : La construction du sens commun stratégique, Peter Lang, Bruxelles, 2010, p. 22.

21. Wasinski C., «Raconter la guerre techniquement : rendre la guerre émotionnellement possible », Res Militaris, 2-2, 2012, p. 1.

22. Wasinki C., op. cit., p. 205.

23. Foucault M., Surveiller et punir : Naissance de la prison, Gallimard, Paris, 1975, p. 34. 
lequel s'exerce un pouvoir de légitimation d'une certaine forme de violence qui permet au final l'extraction du savoir de sécurité.

Il paraît ainsi pertinent d'opérer un retour à un des fondements de la logique du déploiement de la violence de guerre pour comprendre sa mise en légitimité, à savoir l'inscription de cette violence sur les corps. À cet égard, Christine Sylvester souligne l'importance « de s'intéresser à l'un des éléments clefs de la guerre : sa mission de blesser les corps $24 »$. Dans sa lignée, il nous paraît souhaitable de réintroduire cette plus petite unité sur laquelle la violence militaire exerce sa force, le corps humain. La signification, l'ordonnancement du corps dans le déploiement de la coercition conditionne sa mise en acceptabilité, sa mise en sens (il)légitime. Selon Caroline Holmqvist, la virtualisation de plus en plus poussée de la guerre aurait amené un changement de la vision du corps dans la guerre, qui se traduirait par une « dissolution entre le corporel et l'incorporel $25 »$. La légitimité de la guerre par le technologique ne se fait pas uniquement par la disparition du corporel, mais plutôt par sa mise en lien, son va-et-vient avec l'incorporel. La violence est effectivement exercée et se déploie dans la réalité sur les corps ${ }^{26}$, ceci fondant l'ontologie même de la guerre, mais cette réalité de la chair est replacée dans une perspective incorporelle de disparition qui place la guerre dans le moule de l'acceptable et du légitime. Cette « intercorporalité 27 » constitue donc un espace de négociation, d'autorisation développé au travers des technologies et au sein duquel et par lequel s'exerce le pouvoir de légitimation de la force militaire.

Ce procédé dynamique de négociation du facteur humain dans la guerre s'effectue ainsi principalement par l'emploi de technologies militaires particulièrement perfectionnées ${ }^{28}$. James Der Derian s'est précisément penché sur la manière dont la technologie militaire pouvait rendre la guerre plus acceptable. S'interrogeant sur la virtualisation des moyens de faire la guerre, Der Derian en vient à la conclusion que ces nouvelles pratiques contribuent à faire de la guerre un «spectacle virtuel qui n'est pas si immédiatement sanglant que le théâtre "réel" de la guerre et qui, dans son pur excès, apparaît souvent plus comique que tragique 29 ». La distanciation par rapport à l'acte réel de violence

24. Sylvester C., "War Experiences/War Practices/War Theory", Millennium - Journal of International Studies, 40-3, 2012, p. 484.

25. Holmqvist C., "Undoing war: War Ontologies and the Materiality of Drone Warfare", Millennium - Journal of International Studies, 41-3, 2013, p. 14.

26. Pour une lecture dédiée à cette expérience corporelle de la guerre, on lira avec intérêt : McSorley K., War and the body: Militarisation, Practice and Experience, Routledge, London, 2012. Pour l'auteur, la guerre serait une " pratique sociale intégrée corporellement " (p. 236). Il met l'accent sur trois implications de la manipulation du corps dans la faisabilité de la guerre : 1) la préparation du corps à la guerre (Militarizing Bodies), 2) l'expérience corporelle directe et sensorielle du corps dans la guerre (Embodying War), 3) la gestion éthique et politique de la souffrance des corps après la période de guerre (Corporeal Aftermaths).

27. Holmqvist C., loc. cit., p. 13.

28. Angelini L., Wasinski C., "Raisonnement stratégique et construction des énonciateurs légitimes ", Res Militaris, 3-1, 2012, p. 11.

29. Der Derian J., Virtuous War: Mapping the Military-Industrial Media-Entertainment 
qui s'effectue par la médiation des moyens informatiques, tels les écrans, les logiciels de ciblage ou les simulateurs, rend la violence plus acceptable et facile à mettre en œuvre ${ }^{30}$. La conséquence physique de l'emploi de la violence n'est plus directement vécue, la virtualisation du champ de bataille faisant de la guerre un événement à la limite de l'anodin et du jeu vidéo ${ }^{31}$.

Dans un autre registre, Hugh Gusterson démontra comment la virtualisation de plus en plus poussée du vécu de l'arme nucléaire a contribué à faire de cette arme d'une puissance phénoménale un instrument généralement accepté. Selon l'auteur, la mise en données chiffrées de l'arme nucléaire ${ }^{32}$, ainsi que la simulation de plus en plus fréquente de son potentiel destructeur à partir de calculs informatiques ${ }^{33}$, a contribué à provoquer une disparition esthétique des souffrances humaines susceptibles d'être générées par l'explosion de cette arme. Hugh Gusterson parle de « discours techno-stratégique [...] caractérisé par son manque d'émotion, son modèle de la théorie des jeux [...], son penchant pour l'abstraction [...], sa concentration sur les logiciels plus que sur les gens, et son affirmation fondamentale, inquestionnée et inquestionnable selon laquelle le développement des armes nucléaires doit continuer 34 ». En bref, et pour reprendre la subtile formule de Carol Cohn, cette déshumanisation de la violence guerrière permet de et autorise à «penser l'impensable » 35 .

\section{Le cas de l'intervention militaire au-dessus de la Libye : l'(in)sécurisation par l'hyper-rationalisation de la guerre}

Dès les premiers instants de l'opération, l'OTAN opère le portrait de l'Autre sur la base d'un vocabulaire militaire autour duquel est en question la rationalité d'emploi de la violence. Le but affiché dans les discours produits par l'OTAN est de détruire de manière précise toute force présentant une menace pour les civils tout en évitant de les toucher : "Nous frappons avec attention et précision pour maximiser l'effet de nos actions, tout en minimisant le danger pour les civils. C'est en opposition absolue avec les forces proKadhafi, qui assiègent leurs propres villes et bombardent les centres villes. Les troupes de Kadhafi cachent des chars dans les centres villes, à proximité d'écoles et de mosquées. C'est complètement irresponsable 36 ». Cette déclaration est très représentative du mécanisme $d$ '(in)sécurisation mis en place par l'OTAN, à savoir une entrée en opposition avec son emploi limité et précis de la violence avec celui de Kadhafi.

Network, Routledge, New York, 2009, p. 112.

30. Ibid., p. 165.

31. L'auteur évoque en effet l'utilisation de jeux vidéos par l'armée américaine pour entrainer les soldats à tirer. Der Derian cite notamment la reprise du jeu Doom par les Marines (p. 89).

32. Gusterson H., Nuclear rites: a weapons laboratory at the end of the Cold war, University of California Press, Berkeley, 1996, p. 108.

33. Ibid., p. 109.

34. Ibid., p. 205.

35. Cohn C., "Sex and Death in the Rational World of Defense Intellectuals", Signs, 12-4, 1987, p. 709. 
Cette différenciation s'opère tout d'abord à travers l'emploi des logiciels de ciblage. Un premier logiciel de traitement des données était employé : le Joint targeting system (JTS). Il s'agit du support à partir duquel les dossiers de ciblage étaient constitués. Il contient des vidéos, textes, photographies, cartes, etc., de cibles potentielles qui servaient à la constitution des dossiers de ciblage ${ }^{37}$. L'équipage ou le drone qui survolait un objectif envoyait les données à la salle d'opération. Celles-ci étaient ensuite collectées dans le JTS ${ }^{38}$. Il contenait donc les cibles qui ont été frappées, qui allaient être frappées, qui étaient susceptibles d'être frappées en cas d'actions perçues comme hostiles contre les civils.

Le logiciel de ciblage Integrated command and control software for air operation (ICC) entre ensuite en jeu. Il s'agit d'un «environnement intégré de Commandement, de Contrôle, de Communications et de Renseignement/Information (C3I2) qui fournit un soutien à la gestion de l'information et à la décision lors d'opérations aériennes de l'OTAN en période de paix, d'exercice et de guerre. L'ICC fournit un soutien pour les fonctions critiques de contrôle et de commandement aérien (C2) à la composante air du commandement et au niveau du Centre de commandement des opérations aériennes (CAOC) ${ }^{39}$ ». Il s'agit plus concrètement d'un logiciel de ciblage qui permet d'agir au moment d'actions multinationales. L'ICC est une plateforme informatique/cartographique commune autour de laquelle s'organise de façon virtuelle la désignation des cibles. Il fonctionne sur la base du système Google map, et contient des calques cartographiques constitués à partir de photographies ou de cartes. Afin de cibler un objectif à engager, les données contenues sur une cible dans le JTS sont positionnées sur les cartes du logiciel ICC. Dès lors visible sur les écrans, la cible est prête à être sélectionnée, les avions envoyés et l'objectif frappé.

Si l'interaction de base entre logiciels reste la même, le procédé de décision des bombardements peut se manifester sous deux formes. Le premier est le deliberate targeting. Il s'agit de frappes décidées à l'avance et qui font l'objet d'un traitement sous 24 à 48 heures : « Les officiers de renseignement repèrent une cible, l'étudient, montent un dossier d'objectif, l'objectif est validé par le chef de l'opération avec ses conseillers. Une fois que le dossier est validé, il est ré-envoyé aux escadrons, les pilotes préparent la mission, partent en vol, délivrent leur armement, et reviennent $40 \%$. Le second est le dynamic targeting. Il

36. Rasmussen A. F., NATO Secretary General's Monthly press conference, 11 April 2011.

37. Entretien. Anonymat demandé.

38. Ibid.

39. Nato programming center, NATO-wide Integrated Command and Control Software for Air Operations (ICC), Disponible sur : http://www.npc.nato.int/htm/icc.htm (Consulté le 14/01/2013). 
s'agit « d'un engagement en temps réel sur des cibles qui sont estimées menaçantes dans l'immédiat et qui ne peuvent attendre un traitement plus long ${ }^{41}$ ».

L'évaluation des dommages collatéraux s'effectuait également par l'emploi d'un logiciel. Chaque fois qu'une cible pouvait potentiellement être engagée, « un sous-officier du CAOC rentrait les coordonnées dans le logiciel approprié, qui effectuait un traçage de cercles de distance autour du point d'impact de la bombe. Ces cercles concentriques projetaient une évaluation du niveau des dommages collatéraux en fonction de leur plus ou moins grande proximité du point d'impact 42 ». Le logiciel se nomme Fast Assessment strike tool-Collateral damages (FAST-CD) ou « Bugsplat » : «Un programme du JWAC (Joint Warfare Analysis Center) qui permet une évaluation détaillée des possibles dommages collatéraux d'une attaque et des moyens d'en réduire ou diminuer les effets 43 ». Cette méthodologie d'évaluation issue de la mise en séries statistiques et des probabilités chiffrées des risques de dommages collatéraux émanant du logiciel a pour résultat final la naissance d'un système de notes allant de 1 à $5{ }^{44}$. Le niveau 1 désigne le risque le plus bas de dommages collatéraux et le niveau 5 le risque le plus élevé. En fonction du niveau de risque, le personnel habilité à donner l'autorisation de tir est plus ou moins élevé dans la hiérarchie : «De 1 à 2, on peut imaginer que c'est soit l'équipage qui, de sa propre initiative va tirer en expliquant qu'il y a peu de risques. À 3 ou 4 ce serait le chef de la salle d'opération, et le niveau 5 relèverait du Général qui commande l'opération 45 ».

La précision de ces outils et les procédés routinisés de décision qui y sont associés permirent très concrètement de rendre possible cette distinction entre l'emploi de la force par Kadhafi et celui de l'OTAN. Tout paraissait calculé, mis en chiffres, rationalisé, le risque d'erreur tendait à être évacué au maximum, des gradations hiérarchiques s'opéraient dans la prise de décision en fonction des risques de pertes civiles. Tout était mis en œuvre pour les éviter, par opposition à Kadhafi qui était représenté comme les provoquant intentionnellement. Le procédé d'(in)sécurisation qui émane de ces logiciels s'inscrit dans une double dynamique de la gestion des corps humains à protéger : individualisation/classification (généralisation) de l'objet « corps ». D'un côté, chaque vie compte et doit être préservée, de l'autre, les corps apparaissent dans ces logiciels comme des ensembles abstraits et chiffrés. Loin d'être contradictoires, ces éléments sont les deux parties d'un même processus d'inscription d'un pouvoir lié à la définition de la sécurité sur les corps. Pour préserver la vie de chacun, les moyens doivent être rationalisés, pour être toujours plus

40. Entretien. Anonymat demandé.

41. Ibid.

42. Ibid.

43. LtCol Buck Larkin D.B., "Weaponeering anyone?", The Air, Land, Sea Bulletin, 1, 2005, p. 11.

44. Entretien. Anonymat demandé.

45. Ibid. 
efficaces et précis, ce qui exige une classification plus large de l'objet (le corps) du savoir protecteur. La diversité, l'individualité est inscrite dans une généralité d'ordre technologique, pour qu'elle soit préservée. La même logique était appliquée à Kadhafi, mais sous l'angle de la destruction. Dans la rhétorique otanienne, Kadhafi détruisait en masse, l'individu ou la diversité n'étaient pas protégés, la mort fut donnée de manière illimitée, selon une logique de classification indiscriminée qui assimilait l'individu à la population entière : la diversité était inscrite dans la généralité, pour qu'elle soit détruite. À cet égard, les représentants des trois principaux États participant à l'intervention au sein de l'OTAN n'auront eu de cesse d'évoquer l'aspect indiscriminé de la violence employée par l'ancien chef d'État libyen. Tandis que François Fillon se déclara « horrifié par ce déferlement de violence 46 », Hillary Clinton parla de "bain de sang [...] inacceptable 47 ». Barack Obama évoqua quant à lui une violence « scandaleuse et inacceptable 48 ». Gene Cretz, Ambassadeur américain en Libye, parla même "d'actions et d'attaques barbares » dans un discours à la Maison Blanche ${ }^{49}$. La rationalisation de la violence s'opérait d'autant plus que ces logiciels tendent à faire de la guerre un objet déshumanisé, car l'acte de violence s'effectuait par le biais virtuel des écrans informatiques, de données chiffrées émanant de logiciels électroniques, qui aseptisent la réalité physique des bombardements. Ceci permit ainsi de rendre l'image d'une guerre rationnellement menée en opposition à un emploi démesuré de la violence par Kadhafi.

\section{Les échanges d'informations militaires}

Les échanges d'informations militaires constituaient une seconde catégorie technologique permettant une forte rationalisation de l'action militaire. Commençons par le système de chat'. Il s'agissait du moyen de communication employé à l'intérieur du CAOC : «Les membres de la salle d'opération s'échangeaient les informations par le biais de systèmes de chat' type MSN. Cela permettait un échange rapide et aussi de conserver les conversations si l'on souhaitait y revenir plus tard afin de savoir ce qui a été entrepris par le passé. Les coordonnées des cibles apparaissaient également sur les chat' avant d'être rentrées dans le système JTS 50 ». Certains AWACS étaient également en possession de matériel qui leur permettait de recevoir le chat'. Ces derniers pouvaient « ainsi recevoir les informations du CAOC par free text (chat'), ce qui avait l'avantage d'éviter les mauvaises compréhensions à la radio 51 ».

46. Fillon F., cité dans Le Monde, "Kadhafi lâché par certains de ses diplomates ", 22 février 2011.

47. Clinton H., cité dans Barthet E., «Tout le monde attend la chute de Tripoli », Le Monde, 28 février 2011.

48. Obama B., cité dans Le Monde, «Le régime libyen perd le contrôle de l'Est », 24 février 2011.

49. Gertz G., Current events in Libya, Washington D.C., 25 March 2011.

50. Entretien. Anonymat demandé.

51. Ibid. 
Evoquons également le cas particulier de la Liaison 16 (L16) : il s'agit du système sécurisé de partage d'informations de l'OTAN qui « soutient l'échange de données tactiques essentielles à la coordination des unités et des plates-formes [...] militaires. Les données recueillies par les différents capteurs disséminés sur la zone de combat [...] sont fusionnées par la Liaison 16 qui élabore une "image” cohérente et actualisée de la situation de terrain. Cette représentation tactique est diffusée vers les différents acteurs impliqués dans l'opération [...] 52 ». Les données que s'échangent les pilotes des avions concernent « la position des forces amies et ennemies, le niveau de carburant, l'armement disponible, les conditions météorologiques, les coordonnées de cibles [... $]^{53}$ ». En d'autres termes, la L16 consiste en une production d'imagerie à distance du champ de bataille qui est ensuite partagée par l'ensemble des acteurs de l'opération et à partir de laquelle la réalité est interprétée. La L16 est très utilisée par l'OTAN car « la représentation visuelle proposée sur les écrans soulage les pilotes de fastidieux calculs : les symboles et les couleurs sont non ambigus et facilitent la compréhension de la situation. [...]. Un pilote précise : “avec la Liaison 16, vous n’avez plus à vous construire une représentation mentale de la situation tactique. Le système le fait pour vous ! Du coup, vous pouvez donner beaucoup de vos capacités intellectuelles sur le plan tactique, vous êtes beaucoup moins saturé” 54 ». Parfois même, « les données peuvent être envoyées directement dans le système d'arme de l'avion. Avec un avion comme le Rafale équipé de la L16, il était possible de lui envoyer les coordonnées de la cible, qui apparaissaient sur l'écran du pilote. Ce dernier n'avait plus qu'à sélectionner la cible, et automatiquement les coordonnées entraient dans le missile et le missile partait. Il s'agit du digital targeting 55 ».

Enfin, deux outils très perfectionnés permettaient de récupérer des informations sur les cibles potentielles : les drones et les renseignements d'origine électromagnétique (ROEM). Du fait de l'absence officielle de troupes au sol 56, les premiers fournissaient une imagerie aérienne du terrain. Le second outil est beaucoup plus élaboré. Lorsque l'imagerie aérienne ne permettait pas de discerner la nature civile ou militaire d'un bâtiment, « les membres du renseignement décryptaient les activités électromagnétiques (radio, communications électroniques) qui en émanaient grâce à des capteurs présents sur le territoire. Cela permettait de localiser et de frapper les PC ou les QG. Sur un cas particulier, nous avions remarqué grâce à ces décryptages qu'un observateur d'artillerie des forces pro-Kadhafi donnait des indications aux tireurs. On savait donc qu'il s'agissait d'instructions de tirs. On a alors engagé la position d'observation d'artillerie 57 ».

52. Gode C., Hauch V., Lasou M., Lebraty J.F., « Une singularité dans l'aide à la décision : le cas de la Liaison 16 », Systèmes d'Information et Management, 2-17, p. 16.

53. Ibidem.

54. Ibid., p. 22.

55. Entretien. Anonymat demandé.

56. Il a en effet été révélé que des petits groupes de commandos ont été envoyés sur le terrain pour aider à la désignation des cibles. 
A nouveau, la rationalité est très présente dans l'échange d'informations. Conséquences en termes de représentation ? La distinction entre "preuve légale » 58 et « preuve codifiée » dans les justifications des cibles à détruire. $\mathrm{La}$ première renvoie à l'appréciation subjective de la véracité de la preuve par celui qui juge 59 et la seconde, à l'appréciation objective par référence à un code qui établit la qualité de la preuve. L'OTAN paraissait se situer dans la deuxième perspective. Ces réseaux d'informations ultra-perfectionnés tendent à établir une véracité de la preuve du statut menaçant d'une cible proche de la vérité codifiée. La preuve est établie selon des procédés de codifications technologiques avant l'acte de destruction, la subjectivité du jugement de l'information tend à être écarté au profit d'une confiance dans les données recueillies de manière machinale par les technologies. Preuve est faite objectivement, destruction est faite rationnellement. A l'inverse, Kadhafi fut dépeint comme se situant dans le schéma de la preuve légale : il paraissait détruire sans même savoir si preuve était faite du caractère justifié de l'acte de violence. Dans le premier cas, le traitement de la preuve est inscrit dans des processus complexes de rationalisation technologique objective, faisant de l'effet destructeur une opération légitime car fixée sur une justification empiriquement "vraie ». Dans le second, la preuve est laissée à l'appréciation subjective, établissant l'acte de violence sur une base incertaine, injustifiée, et l'inscrivant de surcroît dans la folie dépeinte de Kadhafi. L'informatisation très poussée des modes de communications supprimait par ailleurs tout élément humain dans le partage des données, à tel point que certaines d'entres elles n'eurent même plus besoin d'être saisies manuellement, se programmant directement dans le système d'armement des avions (cas du Rafale avec la L16). Rapidité et précision paraissent les leitmotivs de l'usage de ces moyens de communication, si bien que les militaires admettaient la véracité des données recueillies à travers une réalité représentée sur les écrans et au son des micros. Une représentation de la guerre bien éloignée de la trivialité guerrière associée à Kadhafi...

\section{Les systèmes d'armement: l'exemple des precision-guided munitions (PGMs)}

Le troisième support qui permit à l'OTAN de faire valoir son usage instrumental-rationnel de la violence est l'emploi des precision-guided munitions (PGMs). Il s'agit d'armements guidés qui permettent de frapper une cible tout en évitant au maximum les dommages collatéraux ${ }^{60}$. Ils sont employés dans l'optique de concilier "efficacité militaire " et réduction des pertes civiles ${ }^{61}$. Selon Marshall Beier, le développement des PGMs a participé à la solidification de l'impératif de discrimination entre civils et militaires lors de la pratique

57. Ibid.

58. Foucault M., op. cit., 1975, p. 95.

59. Ibidem.

60. Ward T., The ethics of destruction: norms and force in international relations, Cornell University Press, Ithaca, 2011, p. 163.

61. Wheeler N., “Dying for 'Enduring Freedom': Accepting Responsability for Civilian Casualties in the War against Terrorism”, International Relations, 16-2, 2002, p. 210. 
des bombardements ${ }^{62}$. Si bien que, selon lui, l'utilisation de plus en plus grande de ce type d'armement a consolidé l'idée selon laquelle la distinction entre civils et militaires « est devenue une marque sociale [...] et civilisationnelle ("seuls les sauvages se livrent à des guerres indiscriminées ») de prétentions à la supériorité 63 ». L'emploi des munitions guidées est donc également à mettre en rapport avec des objectifs politiques, qui tendent à fixer une représentation entre ceux dont l'emploi de la violence parait légitime et ceux aux mains desquels elle est perçue comme excessive et injustifiée. Maja Zehfuss en vient même à dire que « la focalisation sur les armements de précision est cruciale dans la représentation de la Western warfare comme éthique et supérieure 64 ».

La majorité des armements délivrés par l'OTAN en Libye furent des PGMs ${ }^{65}$. Comme le montre cette déclaration, l'Alliance en fit référence pour justifier la précision de ses actions : «Je peux dire avec une grande certitude que l'équipement et la technologie de l'OTAN font partie des plus sophistiqués et précis dans le monde. Nous utilisons les systèmes [...] les plus précis et nous employons uniquement des smart weapons. Il s'agit de precision-guided munitions munis d'excellents systèmes de guidage 66 ».

L'emploi des PGMs a donc également permit à l'OTAN, au même titre que les logiciels et les modalités de récupération des informations, de créer une représentation de son emploi de la violence comme particulièrement rationnel en opposition à celui de Kadhafi. L'emploi même des armes s'est en effet opéré dans une optique de limitation des pertes civiles, de protection des populations, se différenciant ainsi de la répression kadhafiste, dont l'essence même de sa représentation par l'OTAN se loge dans l'usage indiscriminé de la violence contre les civils.

\section{Le récit et la grammaire de la guerre}

Intéressons-nous à présent à la manière dont la guerre est représentée du point de vue de son récit. Le premier point intéressant à soulever à cet égard est la diffusion de vidéos des bombardements lors des conférences de presse données par l'OTAN. Dans une conférence du 8 avril, Oana Lungescu, porte-

62. Beier M., "Discriminating Tastes: 'Smart' Bombs, Non-Combatants, and Notions of Legitimacy in Warfare”, Security Dialogue, 34-4, 2003, p. 413.

63. Ibid., p. 416.

64. Zehfuss M., "Targeting: Precision and the production of ethics", European Journal of International Relations, 17-3, 2010, p. 559.

65. Entretien. Anonymat demandé. Des munitions non guidées furent en effet délivrées, les bâtiments de la Marine française ayant notamment tiré 3000 obus. Voir : Assemblée nationale, Compte rendu de la Commission de la défense nationale et des forces armées, 12 octobre 2011, p. 11.

66. Bagellini C., Press briefing on Libya, by the NATO Deputy Spokesperson, Carmen Romero and Brigadier General Clandio Gabellini, Chief Operations Officer, Operation Unified Protector and Commander Peter Clarke, 10 May 2011. 
parole de l'OTAN, entendait «montrer [...] un exemple de ce dont parlait l'Amiral Harding lorsqu'il évoquait les chars des forces pro-Kadhafi qui attaquent les civils dans les zones urbaines. Je pense que l'on a un exemple, une vidéo, où vous pourrez voir un char des forces pro-Kadhafi faire feu dans une aire urbaine, en violation claire de la résolution 1973 des Nations unies. Vous pourrez également voir le pilote [de l'OTAN] surveiller attentivement la zone avant de frapper le char. Et vous pourrez aussi constater l'effet de cette frappe sur les autres forces de Kadhafi qui les disperse et empêche leur attaque 67 ».

D'emblée, la diffusion de cette vidéo met au point une opposition entre le mode d'emploi de la violence de l'OTAN et celui de Kadhafi. Cette différenciation se renforce dans la suite des commentaires de la vidéo par la porteparole : "Cela se déroule à Misrata, mais pas seulement. C'est le type d'attaque indiscriminée dans les zones urbaines que les forces pro-Kadhafi mènent dans toute la Libye, dans des villes comme Brega et Ras Lanouf 68 ».

Plus intéressant encore, l'image diffusée du bombardement témoigne d'une représentation éthérée de ses conséquences. En effet, l'image est celle d'une cible en noir et blanc prise d'un avion et lorsque le char en question est frappé, nous ne voyons qu'une explosion, sans voir ni entendre les conséquences réelles du largage de la bombe. Les morts ne sont pas visibles, pas plus que le bruit de l'explosion, de l'impact ou les cris ne sont audibles ${ }^{69}$. Notons au passage que le son a été coupé lors de la diffusion de la vidéo. La distanciation par rapport à l'acte de violence est très forte. Le corps de ceux qui étaient dans le char est meurtri sans vraiment l'être. La réalité triviale de la mort provoquée par ces bombardements est évacuée, pour renforcer encore plus l'opposition entre l'emploi de la violence par l'OTAN et celui de Kadhafi. La violence de l'Alliance suscite une mort déshumanisée, invisible à l'œil et inaudible aux oreilles. De là elle puise une représentation instrumentale-rationnelle de son emploi de la violence par opposition à celui de Kadhafi dépeint comme « outrageant $» 70$.

La diffusion de vidéos des bombardements lors de la conférence de presse du 10 juin est encore plus significative. La parole est clairement donnée à un militaire, Mike Bracken, porte-parole militaire de l'OTAN pour l'opération Unified Protector, qui fait un exposé d'environ quatorze minutes sur la manière dont les bombardements sont menés, vidéos et photos à l'appui. Il introduit son propos par une différenciation nette entre l'OTAN et Kadhafi,

67. Lungescu O., Press briefing on events concerning Libya by Oana Lungescu, the NATO Spokesperson and Rear Admiral Russell Harding, Deputy Commander of the Combined Joint

68. Ibid.

Task Force Operation Unified Protector, 8 April 2011.

69. Pour visionner la vidéo $:$ http://www.youtube.com/watch?v=xeua3Mi4sy4

70. Lungescu O., Press briefing on events related to Libya by LtGen Charles Bouchard, Commander of the NATO military operations in Libya and NATO Spokesperson Oana Lungescu, 28 March 2011. 
à partir du référentiel de la violence et du matériel militaire : «Nous continuons à frapper les structures de commandement de Kadhafi, son équipement militaire, ses structures de renseignements, et ses autres aménagements militaires qui sont actuellement engagés dans une répression brutale de la population civile » 71 . Alors que Kadhafi vise des civils, des êtres vivants, de manière déraisonnée, l'OTAN frappe du matériel, des cibles militaires, dans une optique rationnelle de protection. En commentant la vidéo d'une frappe sur un bâtiment à Tripoli, il explique qu'elle « montre les frappes aériennes de l'OTAN sur le matériel du régime Kadhafi. [...]. Je voudrais rassurer la population de Tripoli que ces frappes ne sont pas dirigées contre elle ou contre la population libyenne. Elles sont conduites contre ceux qui continuent de soutenir le régime Kadhafi, un régime de violence, de propagande outrageante, et de mensonge 72 ".

En plus d'un récit de la violence déshumanisé, l'image étant celle d'un bâtiment qui explose comme un ballon de baudruche sans pour autant contenir une quelconque trace des conséquences humaines ${ }^{73}$, on constate également une différenciation encore plus marquée entre l'OTAN et Kadhafi, dont le portrait semble s'être radicalisé. Une impression qui se confirme lorsque Mike Bracken commente une vidéo montrant la destruction d'un SA8 ${ }^{74}$ en zone habitée : «La position de ce SA8 démontre une volonté de la part de Kadhafi d'opérer dans la communauté civile. L'OTAN prend soin d'éviter les pertes civiles en attendant que la zone soit vidée de sa population avant de frapper 75 ». Après la destruction et le court moment de silence de l'officier pendant lequel elle se déroule, celui-ci conclut très sobrement: «That's the demise of an $S A 8$ »... Loin d'être une simple description technique, la parole du militaire et la mise en avant de la précision otanienne en opposition à la violence kadhafiste est donc porteuse d'une interprétation de la réalité qui crée de véritables mécanismes d'(in)sécurisation.

L'autre mode de récit de la guerre qui permit de créer la différence de légitimité dans les modes d'usage de la violence est la manière de représenter les dommages collatéraux, ou plutôt de les transformer en "accidents ». Selon Patricia Owens, ce glissement syntaxique n'est pas sans conséquence et tend à renforcer le régime de vérité autour de l'usage instrumental-rationnel de la violence. Selon elle, cette manœuvre génère une « approche entrepreneuriale de la guerre 76 », qui fait des dommages collatéraux une question d'ordre technique plus que politique et humain. L'accident relève de l'erreur, il traduit le

71. Bracken M., NATO and Libya: Press briefing, 10 June 2011.

72. Ibid.

73. Pour visionner la vidéo $:$ http://www.youtube.com/watch?v=s-7HiOnYjZw

74. Le SA8 est le code OTAN pour désigner un 9K33 OSA. Il s'agit d'un système de missile solair de courte portée fixé sur un véhicule unique.

75. Bracken M., NATO and Libya: Press briefing, 10 June 2011.

76. Owens P., “Accidents Don't Just Happen: The Liberal Politics of High Technology 'Humanitarian' War', Millenium - Journal of International Studies, 32-3, p. 597. 
non-intentionnel, car un dommage collatéral n'est dû qu'à une « bombe occasionnelle 77 » dans une volonté beaucoup plus affichée de frapper pour détruire rationnellement. Il s'agit d'une erreur d'ordre technique, qu'il faut rectifier par une amélioration technique afin de rendre les «pertes civiles toujours plus prévisibles $78 »$. Le corps des victimes de ces dommages collatéraux s'assimile alors à des " objets 79 », mis en chiffres et traités par le technologique. Non seulement les morts sont finalement évacués par cette hyper-rationalisation de la gestion des dommages collatéraux, renforçant par ce biais l'aspect rationnel de la violence employée, mais elle porte également un sens aigu de l'altérité, qui se fixe sur la logique de l'intentionnel contre le non-intentionnel : «[...] la comparaison avec le “soin” pris par les États libéraux et les tueries indiscriminées des terroristes et régimes voyous - “les blessures et dommages que nous infligeons accidentellement contre celles infligées délibérément" - a été construite de manière si évidente et valide qu'elle se situe hors de tous doutes et questionnements 80 ". L'intentionnel est dès lors associé à l'illégitime et le non-intentionnel au légitime. C'est en ce sens que l'on peut interpréter la transformation par l'OTAN des dommages collatéraux en accidents lors de son opération en Libye. Cette déclaration de l'amiral Giampaolo Di Paola, Président du Comité militaire de l'OTAN, est très significative : "Quand nous avons lancé cette opération, l'impératif était de ne faire aucune victime et de ne provoquer aucun dommage collatéral. Et “aucun”, pour nous tous, signifie “zéro”. Toutefois, “humainement”, la perfection n’existe pas et nul n'est parfait, mais si vous regardez les chiffres, ceux que nous connaissons, pas ceux diffusés par la propagande de Kadhafi, avec le chiffre de seulement un incident pour 10000 frappes, nous pouvons dire, il me semble, que cela correspond vraiment à zéro. C'est aussi près de l'objectif “zéro” que l'on peut "humainement” atteindre en matière de victimes. Ce qui est remarquable 81 ».

On passe du « dommage collatéral » à l' « incident », qui semblait justifier la bonne intention de l'OTAN de ne pas frapper volontairement les civils par opposition à Kadhafi. Ces propos vont beaucoup plus loin, l'incident est assimilé à la perfection. Ces propos montrent que l'OTAN a généré la mort, mais de manière tellement infime, que cette mort paraît disparaître ${ }^{82}$. Ces morts paraissent dès lors justifiées, car non-intentionnelles, et servent même à la justification de la précision de l'OTAN. Le retournement est pour le moins habile : des morts à la base non-acceptables servent à légitimer une capacité

77. Ibid., p. 606.

78. Ibidem.

79. Gregory D., The Colonial present: Afghanistan, Palestine, Iraq, Blackwell, Oxford, 2004, p. 212.

80. Owens P., loc. cit., pp. 607-608.

81. Di Paola G., Discours par l'amiral Giampaolo Di Paola, Président du Comité militaire de l'OTAN à la 57 ième session annuelle de l'Assemblée parlementaire de l'OTAN, Bucarest, 8 octobre 2011.

82. Notons au passage que l'enquête de Human Rights Watch effectuée sur 8 théâtres de bombardement a estimé le niveau de dommages collatéraux à 72 personnes. Voir Human Rights Watch, op. cit., p. 4. 
d'action, et sont donc rendues tolérables. Non seulement la mort et les corps meurtris sont évacués du discours, renforçant l'image instrumentale-rationnelle de la violence otanienne, mais l'altérité s'opère également, et ce, de deux points de vue. Premièrement, et comme relevé tout au long de cet article, l'opposition se crée avec la violence employée par Kadhafi représentée comme visant intentionnellement les civils. Deuxièmement, les chiffres donnés par Kadhafi ne paraissent pas crédibles, ils sont issus d'une "propagande », ce qui ne fait que solidifier la légitimé de la bonne parole stratégique de l'OTAN qui détiendrait les véritables chiffres des pertes civiles.

\section{Permis de tuer et définition de la sécurité}

Pour conclure, notre démonstration aura tenté de pointer du doigt le fait que la narration technique de la guerre et l'utilisation de technologies militaires ne sont pas seulement liées à des objectifs éthiques, mais sont étroitement liées à des enjeux politiques inscrits dans la définition d'un sens particulier de la sécurité. Cette étude se veut une piqûre de rappel pour les analystes de l'OTAN qui semblent trop souvent oublier une caractéristique fondamentale de l'Alliance : l'OTAN reste avant tout un organisme militaire qui est appelé pour employer la violence. En d'autres termes, si l'OTAN s'exécute aussi à protéger, elle est liée de manière consubstantielle à la destruction et, in fine, à la mort. La capacité de l'OTAN à rester un centre clef dans la définition de la sécurité n'est pas liée qu'à sa production discursive, à la production explicite d'une idéologie libérale, mais à des pratiques militaires très concrètes qui lui permettent de donner et gérer la mort d'une manière rationnelle, voire managériale. Une dimension capitale pour une organisation censée représenter les canons politiques de ses membres, qui passent par un usage de la violence limité, instrumental et rationnel dans la quotidienneté de la vie sociale. Le corps soumis à l'acte violent constitue ainsi un « lieu d'extorsion de la vérité » 83 sécuritaire. Il est le support concret sur lequel s'exerce un pouvoir de légitimation qui donne à l'OTAN sa capacité de définition de la sécurité sur la base d'un usage instrumental-rationnel de la violence. Gérer la représentation des souffrances corporelles, donner la mort sans la donner, pratiques et narrations guerrières fondamentalement morbides mais relevant en apparence du spectacle et du non-événement. La capacité de l'OTAN à légitimer sa place dans la gestion des insécurités passe notamment par ces techniques de gestion de la mort, une technologie politique des corps, qui lui donnent la possibilité de projeter un sens de la sécurité en partie fixé sur le référentiel de l'emploi d'une violence limitée.

En bout de réflexion, l'OTAN ne serait-elle pas l'expression paroxystique de la « dissonance ontologique 84 » inhérente à la formulation la plus contemporaine de la modernité lorsque celle-ci se voit confrontée au phénomène

83. Foucault M., op. cit., 1975, p. 53. 
guerre ? Une modernité qui refuse les morts, par souci d'affirmer une image pacifique de son contenu, tout en construisant des moyens toujours plus perfectionnés pour tuer de manière efficace ${ }^{85}$. La bureaucratisation de la coercition et les innovations technologiques qu'elle généra au cours du temps permirent aux entités politiques modernes d'augmenter leur efficacité militaire tout en éloignant toujours plus la réalité du champs de bataille. L'OTAN est sans doute l'expression même d'une tentative de résolution de cette tension: une machinerie militaire pourvue d'une forte capacité destructrice mais qui permet également de faire disparaitre ce dont l'affirmation contemporaine de la modernité a le plus honte : le sang, les morts, en d'autres termes... la réalité de la guerre.

84. Malesevic S., The sociology of war and violence, Cambridge University Press, Cambridge, 2010, p. 119.

85. Ibid., p. 129. 